\title{
Isotopic ages of Lentiira - Kuhmo - Kostomuksha olivine lamproite - Group II kimberlites
}

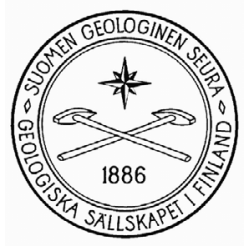

\author{
Hugh O’Brien ${ }^{11 *}$, Dave Phillips ${ }^{2)}$ and Roy Spencer ${ }^{3)}$ \\ 1) Geological Survey of Finland, P.O. Box 96, FI-02151, Espoo, Finland \\ 2) Department of Geology, School of Earth Sciences, University of Melbourne, Victoria 3010, \\ Australia \\ 3) European Diamonds Plc, 22 Grosvenor Square, London W1K 6LF, UK
}

\begin{abstract}
The Lentiira-Kuhmo-Kostomuksha triangle, along the Finland - Russian border and within the central part of the Archean Karelian craton, contains numerous examples of phlogopiterich, ultramafic, mantle-xenocryst-bearing and, in some cases, diamond-bearing dike rocks. Laser probe Ar-Ar data on phlogopite from 3 dike rocks on the Finnish side (Lentiira, Kuhmo) all gave ages within error of each other, $1202 \pm 3 \mathrm{Ma}(2 \sigma), 1199 \pm 3 \mathrm{Ma}(2 \sigma)$ and $1204 \pm 4 \mathrm{Ma}(2 \sigma)$ while a fourth sample produced mixed ages. Published Rb-Sr dates on mineralogically and chemically similar dikes from the Russian side (Kostomuksha) are 1232 $\pm 5 \mathrm{Ma}$. The question remains open whether these represent two distinct age populations or whether differences in isotopic system behavior are the reason for the 30 m.y. age difference.
\end{abstract}

Key words: kimberlite, lamproite, phlogopite, biotite, absolute age, Ar-40/Ar-39, Proterozoic, Lentiira, Seitaperä, Kuhmo, Finland, Kostomuksha, Russian Federation

*Corresponding author e-mail: hugh.obrien@gtk.fi

\section{Introduction}

Kimberlites are ultramafic, volatile-charged, incompatible element-rich magmas that represent a mixture of liquid, mantle peridotite and eclogite detritus carried from depth, and typically megacryst suite minerals such as Ti-pyrope, $\mathrm{Mg}$-ilmenite and $\mathrm{Cr}$-diopside. There are two end-member kimberlite types, based on examples from South Africa: Group I with abundant large, rounded grains (macrocrysts) of olivine, in a matrix of euhderal olivine, monticellite, perovskite, magnesian ulvöspinel-magnetite, Ba-rich phlogopitekinoshitalite mica, calcite and serpentine, and Group
II typically with abundant phlogopite \pm olivine in a matrix of phlogopite, K-richterite and other diagnostic minerals (Table 1). Olivine lamproites show some similarities to Group II kimberlites but exist, for example, in W. Australia (Argyle diamond mine), Montana and Wyoming USA, and southern Spain, whereas no rocks absolutely identical to Group II kimberlites have been found outside of southern Africa.

In the area of Lentiira and Kuhmo, in eastern Finland (Fig. 1), occur a series of rocks with characteristics of both olivine lamproites and Group II kimber- 


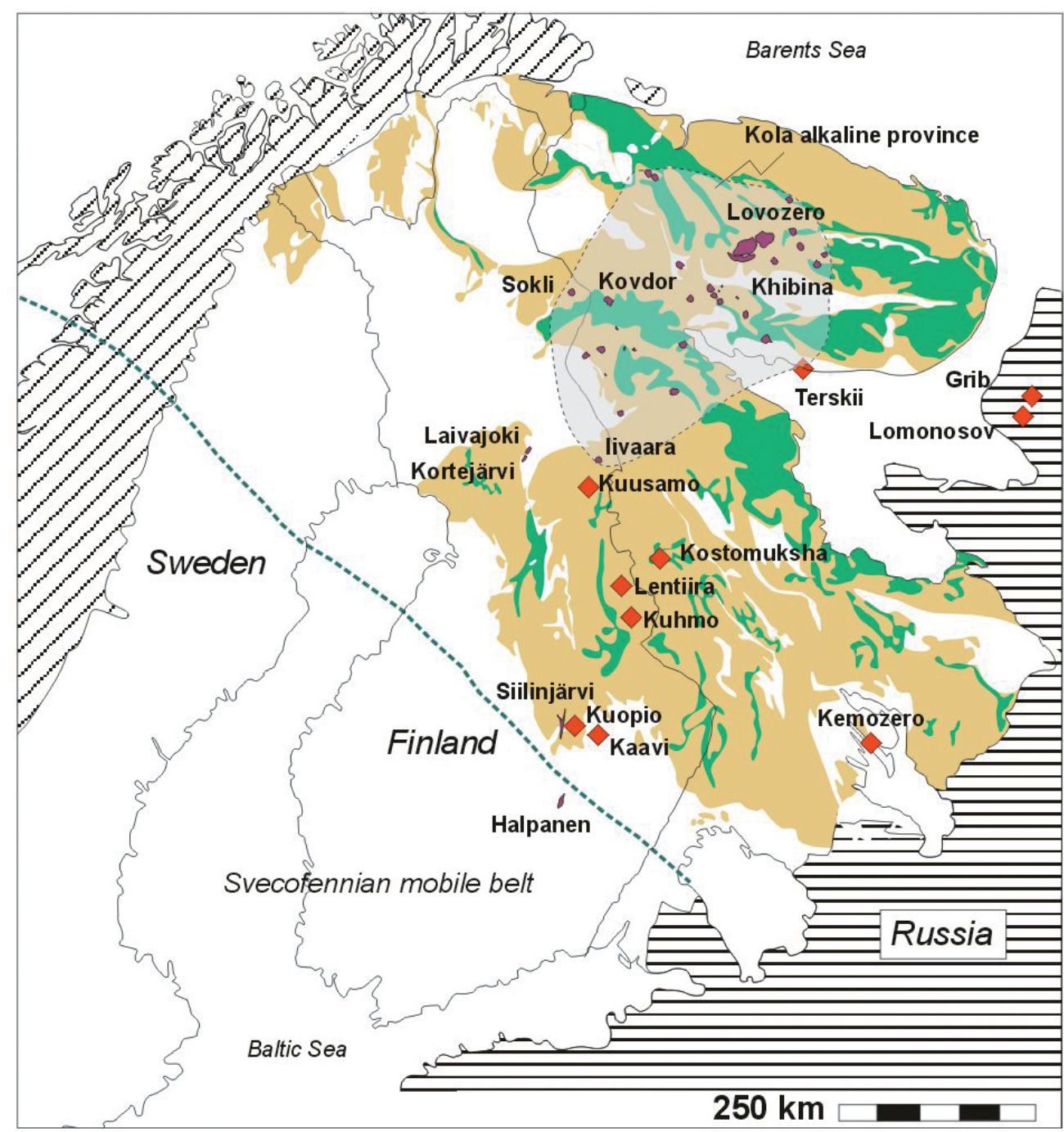

Fig. I. Map showing the Lentiira-Kuhmo-Kostomuksha kimberlite/lamproite locations (black diamonds) within the Archean Karelian craton (SW margin marked by dashed line) along with other kimberlitic occurrences in the Fennoscandian shield.

lites (O'Brien \& Tyni, 1999), although in fact they share more characteristics with the latter. The most notable feature of these ultramafic rocks is that they contain abundant phlogopite as phenocrysts, and more rarely as macrocrysts. Additionally, they range from being olivine macrocryst-poor to extremely olivine macrocryst-rich, and in some cases contain abundant microxenoliths of mantle peridotite. Additional minerals include K-richterite, Mn-rich ilmenite, Cr-rich spinel zoned to Ti-magnetite, apatite and perovskite in a calcite + serpentine matrix.

In the Kostomuksha region of Russian Karelia, 
about $40 \mathrm{~km} \mathrm{NE}$ of the Finnish occurrences (Fig. 1), a suite of rocks ranging from leucite lamproite to olivine lamproite to Group II kimberlites has been identified and studied. These rocks have been termed K2L by Mahotkin (1998), in reference to their intermediate mineralogies between Group II kimberlite and olivine lamproite. The occurrence of leucite (now pseudoleucite), priderite and the lack of matrix serpentine in many of these rocks indicate that they are closer to true lamproites, yet many contain groundmass carbonate, rare to absent in typical lamproites.

All of the rocks discussed here are phlogopite-rich, but differ in that those from the Finnish side of the border contain groundmass carbonate and serpentine and have perovskite rather than priderite, all characteristics more typical of Group II kimberlite. Detailed research into whether there is a true E-W variation in magma types is the topic of another study, but here we report age evidence that may indicate a temporal separation of the Kostomuksha and Lentiira-Kuhmo kimberlite/lamproite dike fields by a few tens of millions of years. However, it is also important to note that regardless of distinct age populations and detailed mineralogical differences, on a broader scale there is a general mineralogical, geochemical and isotopic similarity among the lamproite and Group II magmas from Lentiira-Kuhmo-Kostomuksha and with the diamondiferous kimberlites of the Arkhangelsk area of Russia; all linked by the fact that they occur within the same Karelian-Kola-Kuloi mega-craton.

\section{Previous Age Data}

Only limited age-dates of the rocks discussed here have so far been published, and it is the goal of this short communication to update the situation with the latest geochronological results on these rocks. Rather imprecise perovskite U-Pb data by ion microprobe from Seitaperä, near Kuhmo can be interpreted to represent an age of around 1.2 Ga (O’Brien et al., 2005) although there is very large scatter in the data. From the Kostomuksha field, a Rb-Sr mineral isochron age of $1232 \pm 4.5 \mathrm{Ma}$ was reported for 4 selected mineral fractions ( 2 phlogopites and 2 pyroxenes) from 2 different dike hand samples (Belyatsii et al., 1997; Nikitina et. al, 1999). Although the data from the entire set of 11 analyses (mineral separates and whole rocks) from 5 different samples show considerably more scatter, recalculating using the entire dataset does not change the outcome significantly because of the very radiogenic $\mathrm{Sr}$ in the two phlogopite fractions. The full dataset gives $1232 \pm 10 \mathrm{Ma}$ with an MSWD of 35 using $0.5 \%$ and $0.05 \%$ std errors for ${ }^{87} \mathrm{Rb} /{ }^{86} \mathrm{Sr}$ and ${ }^{87} \mathrm{Sr} /{ }^{86} \mathrm{Sr}$, respectively. The same two papers (op. cit.) also report a $\mathrm{Sm}-\mathrm{Nd}$ isochron from these samples, $1234 \pm 80$, but the large errors do not help narrow down the time of dike emplacement.

As part of a study of noble gases on the Kostomuksha lamproitic dike rocks, Wiersberg (2001) obtained $\mathrm{K}-\mathrm{Ar}$ ages from what is apparently the same sample suite as studied by Belyatsky et al., 1997 and Nikitina et. al, 1999. Wiersberg (2001) reports K-Ar ages ranging from 1150 to $1246 \mathrm{Ma}$ and an overall age of $1193 \pm 20 \mathrm{Ma}$ (although Isoplot [Ludwig, 2000] suggests $1193 \pm 42 \mathrm{Ma}$ at a $95 \%$ confidence level). Earlier statements about a ${ }^{40} \mathrm{Ar} /{ }^{36} \mathrm{Ar}-{ }^{40} \mathrm{~K} /{ }^{36} \mathrm{Ar}$ correlation for 6 of these same K-feldspar/phlogopite separates giving an age of $1223 \pm 13 \mathrm{Ma}$ (Wiersberg et al., 1999; 2000) was apparently reinterpreted as mixing with atmospheric components (Wiersberg, 2001).

\section{Samples and analytical procedures}

Two samples each from the Kuhmo and Lentiira areas (Table 1) were submitted for ${ }^{40} \mathrm{Ar} /{ }^{39} \mathrm{Ar}$ laser probe analyses of phlogopite to the Noble Gas Laboratory at the University of Melbourne.

The Kuhmo samples comprise: 1. Sample $\mathbf{1 6 . 1 2}$ $\mathbf{2 7} \mathbf{m}$ is a reddish brown rock, composed of approximately $60 \%$ phlogopite (Fig. 2), taken from the original Malmikaivos drill core from hole 12, at $27 \mathrm{~m}$ depth. A full mineralogical description and microprobe data for this rock are given in O'Brien \& Tyni (1999). 2. Sample 6501-B-2 was taken by trenching where the till is relatively thin at the NW corner of the Seitaperä intrusion as part of a Ph. D. thesis project (Lehtonen, 2005). The sample is considerably 


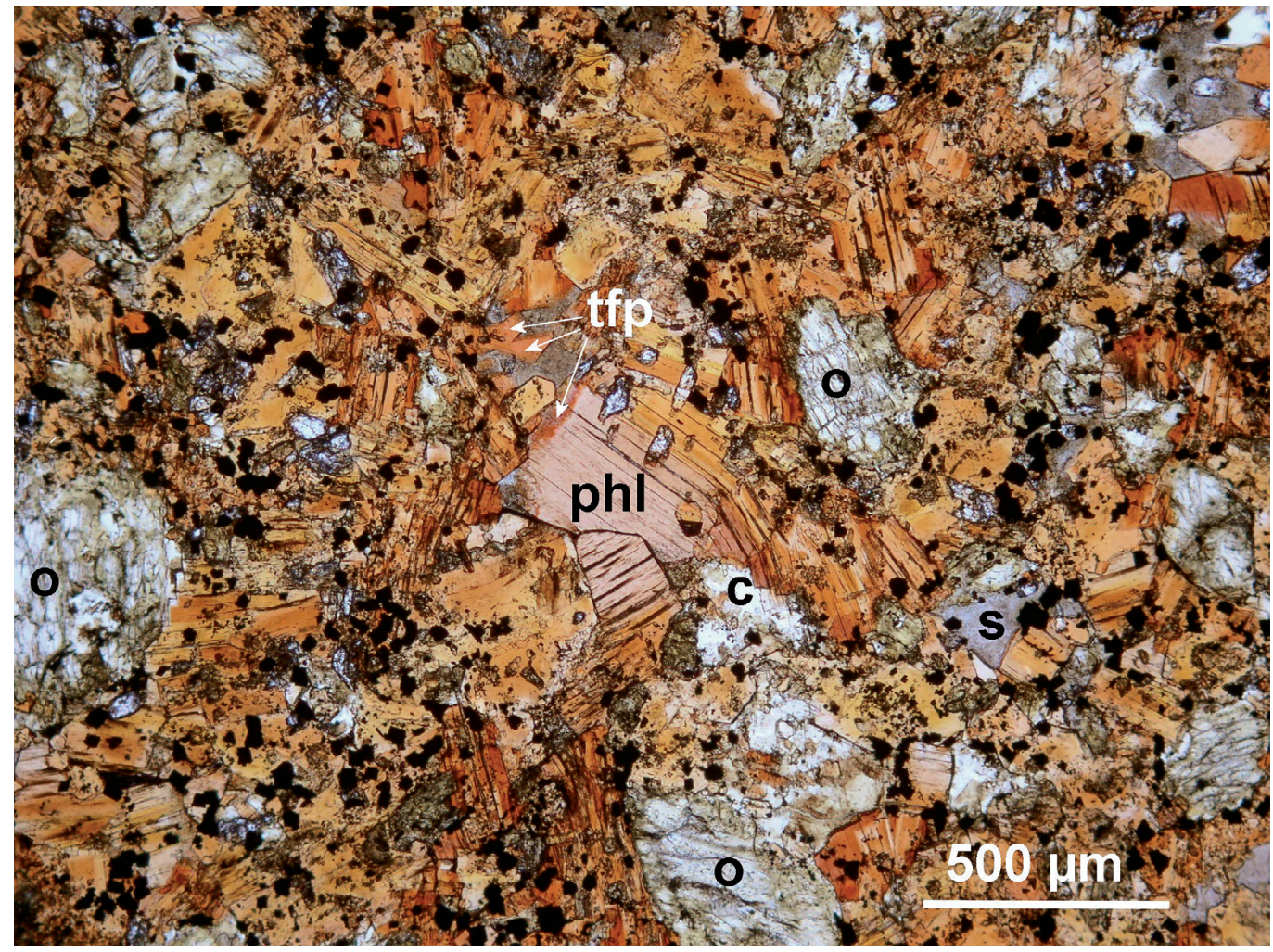

Fig. 2. Photomicrograph of Seitaperä sample $16.12,27 \mathrm{~m}$. Rock is dominantly phlogopite (phl), zoned at margins to tetraferriphlogopite (tfp), olivine pseudomorphs (o), small black cubes of perovskite \& oxides, carbonate (c), and serpentine (s). Plane polarized light.

coarser than $16.1227 \mathrm{~m}$, yet contains a similar modal percentage of fresh, red-brown phlogopite, making it an ideal sample for Ar-Ar dating.

The Lentiira samples comprise: 1 . Sample LentW1 is from a roughly $1 \mathrm{~m}$ wide dike at the western extent of the Lentiira Group II kimberlite field. The sample was slightly more altered than the Seitaperä samples, and some alteration of phlogopite is apparent. The main reason for choosing the sample is the abundance of mantle xenocrysts (particularly pyrope) that it contains. 2. Sample DC401 represents a mixed sample containing crushed material from a somewhat altered kimberlitic rock and the surrounding country rocks. Many of the grains in the sample were dark brown to black representing biotite flakes from the granitic bedrock so a mixed age for this sample was anticipated.
Phlogopite mineral separates were prepared from each sample using standard crushing, sieving, desliming and electromagnetic methods. Approximately 20 - 30 phlogopite grains were handpicked from each sample and washed in deionized water and acetone prior to being shipped for irradiation. The grains were wrapped in aluminum packets and placed into an aluminum irradiation canister together with aliquots of the flux monitor GA1550 (Age = $98.8 \pm 0.5$ Ma; Renne et al., 1998). Packets containing $\mathrm{K}_{2} \mathrm{SO}_{4}$ were placed at either end of the canister to monitor ${ }^{40} \mathrm{Ar}$ production from potassium. The irradiation canister was irradiated in position 5C of the McMaster reactor, Hamilton, Ontario, Canada.

After irradiation, the samples were removed from their packaging and individual phlogopite grains were loaded into a copper sample holder. The sam- 
Table I. Lentiira and Kuhmo samples submitted for $40 \mathrm{Ar} / 39 \mathrm{Ar}$ dating

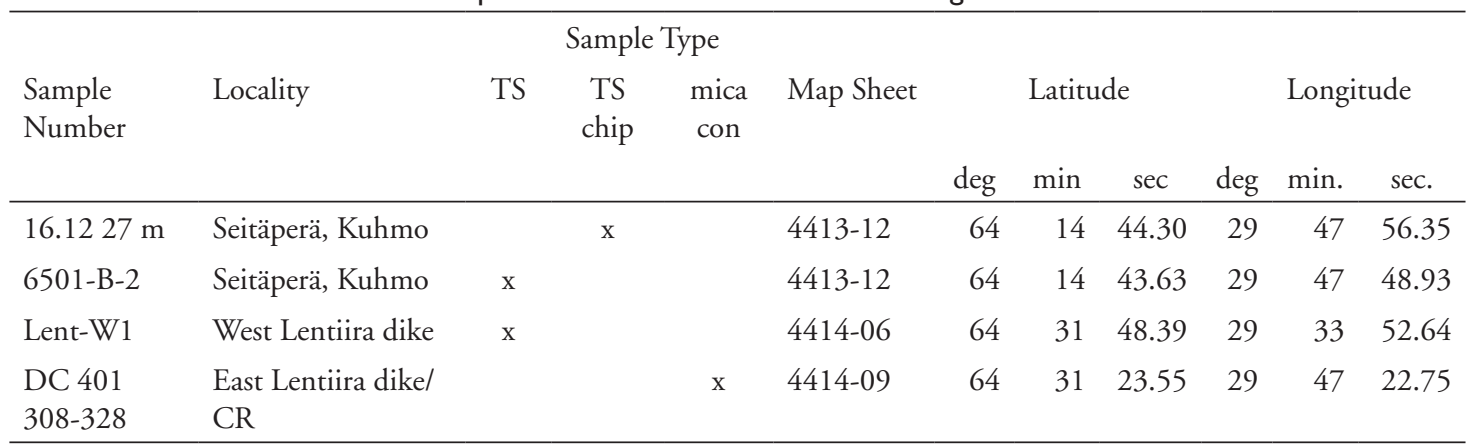

ples were then individually step-heated using a CW Nd:YAG laser. ${ }^{40} \mathrm{Ar} /{ }^{39} \mathrm{Ar}$ analyses were carried out on a MM5400 mass spectrometer using a Daly detector. Mass discrimination was monitored by analyses of standard air volumes. Correction factors for interfering reactions are as follows: $\left({ }^{36} \mathrm{Ar} /{ }^{37} \mathrm{Ar}\right) \mathrm{Ca}=2.63$ $( \pm 0.01) \times 10-4 ;\left({ }^{39} \mathrm{Ar} /{ }^{37} \mathrm{Ar}\right) \mathrm{Ca}=6.86( \pm 0.03) \times 10-4$; $\left({ }^{40} \mathrm{Ar} /{ }^{39} \mathrm{Ar}\right) \mathrm{K}=0.0015( \pm 0.007) . \mathrm{Ca} / \mathrm{K}$ ratios were calculated as follows: $\mathrm{Ca} / \mathrm{K}=1.9 \times{ }^{39} \mathrm{Ar} /{ }^{37} \mathrm{Ar}$. The reported data have been corrected for system backgrounds, mass discrimination, fluence gradients and atmospheric contamination. Unless otherwise stated, errors associated with the age determinations are one-sigma uncertainties and exclude uncertainties in the J-value, age of the fluence monitor GA1550 and the decay constants. Decay constants are those of Steiger \& Jager (1977). The ${ }^{40} \mathrm{Ar} /{ }^{39} \mathrm{Ar}$ dating technique is described in detail by McDougall and Harrison (1999).

\section{Results}

For sample $\mathbf{1 6 . 1 2} 27 \mathrm{~m}$ a total of five single grains were step-heated in $2-4$ increments (Table 2). Most grains are characterized by slightly elevated low temperature ages, possibly caused by release of excess argon from inclusions and/or minor alteration-induced recoil loss of ${ }^{39} \mathrm{Ar}_{\mathrm{K}}$ (Fig. 3a). However, the highest temperature steps are concordant, with a weighted mean age of $1202 \pm 3 \mathrm{Ma}(2 \sigma$, incl. J-error) (Fig. 3b). This result is also within error of the plateau age determined for grain \#5 (1200 $\pm 7 \mathrm{Ma} ; 2 \sigma$, incl. J-er- ror) (Fig. 3a).

Five mica grains from Sample 6501-B-2 were each step-heated in four increments (Table 2). Grains \#1 and \#2 produced somewhat discordant age spectra, but with similar high temperature ages of $-1200 \mathrm{Ma}$ (Table 2). The other three grains are characterized by age plateaus with weighted mean ages of $1209 \pm 8$ $\mathrm{Ma}(2 \sigma$; incl. J-error), $1200 \pm 6 \mathrm{Ma}(2 \sigma$; incl. J-error) and $1199 \pm 8 \mathrm{Ma}(2 \sigma$; incl. J-error) (Figs. 4a-c). The weighted mean result for the high temperature steps from all five grains is $1199 \pm 3 \mathrm{Ma}(2 \sigma$; incl. Jerror) (Fig. 4d).

Six grains from sample Lent-W1 were individually step-heated in two to three increments (Table 2). Five grains are characterized by younger low temperature steps, presumably due to minor ${ }^{40} \mathrm{Ar}$ loss. Grain \#1 exhibits an elevated low temperature age, possibly caused by release of excess argon from inclusions and/ or alteration-induced recoil loss of ${ }^{39} \mathrm{Ar}_{\mathrm{K}}$. The high temperature results are, however, concordant, with a weighted mean age of $1204 \pm 4 \mathrm{Ma}(2 \sigma$, incl. J-error; MSWD = 0.96) (Fig. 5).

A total of eight grains were step-heated from sample DC401. Low temperature apparent ages range from $1173 \pm 11 \mathrm{Ma}$ to $2001 \pm 24 \mathrm{Ma}$, whereas the highest temperature steps are mostly older at $1384 \pm$ $7 \mathrm{Ma}$ to $2443 \pm 57 \mathrm{Ma}$ (Table 2; Fig. 6). As this sample represents a mixture of kimberlitic material and country rock, the spread in ages likely comes from micas from the surrounding rocks that have been variably reset by injection of the dike magma. In this case the youngest ages may represent the closest ap- 
Table 2. ${ }^{40} \mathrm{Ar} /{ }^{39} \mathrm{Ar}$ Step-Heating Analytical Results

\begin{tabular}{|c|c|c|c|c|c|c|c|c|c|c|c|}
\hline $\begin{array}{l}\text { Grain } \\
\text { No }\end{array}$ & $\begin{array}{l}\text { Step } \\
\text { No }\end{array}$ & $\begin{array}{l}\text { Cum } \\
\%^{39} \mathrm{Ar}\end{array}$ & ${ }^{40} \mathrm{Ar} /{ }^{39} \mathrm{Ar}$ & ${ }^{37} \mathrm{Ar} /{ }^{39} \mathrm{Ar}$ & ${ }^{36} \mathrm{Ar} /{ }^{39} \mathrm{Ar}$ & $\begin{array}{l}\text { Vol. }{ }^{39} \mathrm{Ar} \\
\text { x } 10^{-14} \mathrm{~mol}\end{array}$ & $\begin{array}{l}\text { \%Rad. } \\
{ }^{40} \mathrm{Ar}\end{array}$ & $\mathrm{Ca} / \mathrm{K}$ & ${ }^{40} \mathrm{Ar} * / 39 \mathrm{Ar}$ & $\begin{array}{l}\text { Age } \\
(\mathrm{Ma})\end{array}$ & $\begin{array}{c} \pm 1 \text { s.d. } \\
(\mathrm{Ma})\end{array}$ \\
\hline \multicolumn{12}{|c|}{ Seitaperä (Body 16) $(16.12 / 27 \mathrm{~m})$} \\
\hline \multicolumn{12}{|c|}{$\mathrm{J}$-value $=0.0071985 \pm 0.0000072$} \\
\hline \multirow[t]{2}{*}{1} & 1 & 20.0 & 135.4 & 0.0708 & 0.0053 & 2.276 & 98.8 & 0.135 & 133.8 & 1217.3 & 4.3 \\
\hline & 2 & 100.0 & 131.6 & 0.0258 & 0.0013 & 9.336 & 99.7 & 0.049 & 131.3 & 1200.1 & 5.1 \\
\hline \multirow[t]{3}{*}{2} & 1 & 41.6 & 132.9 & 0.1570 & 0.0032 & 2.883 & 99.3 & 0.298 & 132.0 & 1204.8 & 3.9 \\
\hline & 2 & 88.3 & 132.7 & 0.0902 & 0.0017 & 3.246 & 99.6 & 0.171 & 132.2 & 1206.2 & 5.1 \\
\hline & 3 & 100.0 & 133.0 & 0.3210 & 0.0043 & 0.810 & 99.1 & 0.610 & 131.8 & 1203.9 & 8.9 \\
\hline \multirow[t]{3}{*}{3} & 1 & 24.4 & 140.1 & 0.5411 & 0.0080 & 1.789 & 98.3 & 1.030 & 137.8 & 1243.4 & 6.5 \\
\hline & 2 & 43.6 & 133.7 & 0.0109 & 0.0023 & 1.479 & 99.5 & 0.021 & 132.9 & 1211.3 & 7.9 \\
\hline & 3 & 100.0 & 132.2 & 0.0804 & 0.0007 & 4.380 & 99.8 & 0.153 & 132.0 & 1204.9 & 7.1 \\
\hline \multirow[t]{3}{*}{4} & 1 & 36.8 & 134.1 & 0.0425 & 0.0052 & 2.388 & 98.9 & 0.081 & 132.5 & 1208.4 & 4.1 \\
\hline & 2 & 84.4 & 131.7 & 0.0230 & 0.0008 & 3.135 & 99.8 & 0.044 & 131.5 & 1201.6 & 5.4 \\
\hline & 3 & 100.0 & 130.7 & 0.0154 & 0.0002 & 1.040 & 100 & 0.029 & 130.6 & 1195.4 & 5.8 \\
\hline \multirow[t]{4}{*}{5} & 1 & 35.6 & 134.4 & 0.0945 & 0.0040 & 3.666 & 99.1 & 0.180 & 133.3 & 1213.5 & 3.6 \\
\hline & 2 & 72.5 & 131.1 & 0.0198 & 0.0005 & 3.894 & 99.9 & 0.038 & 130.9 & 1197.8 & 3.5 \\
\hline & 3 & 98.2 & 132.7 & 0.0486 & 0.0014 & 2.685 & 99.7 & 0.092 & 132.3 & 1206.7 & 6.7 \\
\hline & 4 & 100.0 & 138.4 & 0.1714 & 0.0015 & 0.176 & 99.7 & 0.326 & 138.0 & 1244.5 & 41.1 \\
\hline \multicolumn{12}{|c|}{ Seitaperä (Body 16) (6501-B-2) } \\
\hline \multicolumn{12}{|c|}{$\mathrm{J}$-value $=0.0071985 \pm 0.0000072$} \\
\hline \multirow[t]{4}{*}{1} & 1 & 35.37 & 130.6 & 0.0078 & 0.0074 & 4.154 & 98.3 & 0.015 & 128.4 & 1180.8 & 3.8 \\
\hline & 2 & 53.14 & 134.2 & 0.0089 & 0.0026 & 2.087 & 99.4 & 0.017 & 133.4 & 1214.4 & 4.9 \\
\hline & 3 & 67.32 & 137.5 & 0.0088 & 0.0064 & 1.665 & 98.6 & 0.017 & 135.6 & 1228.8 & 8.2 \\
\hline & 4 & 100.0 & 132.7 & 0.0154 & 0.0045 & 3.838 & 99.0 & 0.029 & 131.4 & 1200.7 & 4.7 \\
\hline \multirow[t]{4}{*}{2} & 1 & 16.52 & 128.2 & 0.1760 & 0.0055 & 2.221 & 98.7 & 0.334 & 126.6 & 1168.9 & 5.8 \\
\hline & 2 & 40.34 & 133.5 & 0.0196 & 0.0023 & 3.201 & 99.5 & 0.037 & 132.9 & 1210.7 & 7.2 \\
\hline & 3 & 73.94 & 130.9 & 0.0231 & 0.0018 & 4.517 & 99.6 & 0.044 & 130.4 & 1194.1 & 3.3 \\
\hline & 4 & 100.0 & 131.6 & 0.0091 & 0.0020 & 3.503 & 99.6 & 0.017 & 131.0 & 1198.3 & 4.7 \\
\hline \multirow[t]{4}{*}{3} & 1 & 17.47 & 133.5 & 0.0230 & 0.0010 & 1.994 & 99.8 & 0.044 & 133.2 & 1212.9 & 7.4 \\
\hline & 2 & 48.76 & 132.6 & 0.0067 & 0.0016 & 3.573 & 99.6 & 0.013 & 132.1 & 1205.6 & 6.7 \\
\hline & 3 & 82.30 & 132.9 & 0.0099 & 0.0012 & 3.828 & 99.7 & 0.019 & 132.6 & 1208.9 & 6.0 \\
\hline & 4 & 100.0 & 131.6 & 0.0069 & 0.0076 & 2.021 & 98.3 & 0.013 & 129.4 & 1187.3 & 6.9 \\
\hline \multirow[t]{4}{*}{4} & 1 & 4.20 & 52.1 & 0.1393 & 0.0084 & 0.543 & 95.2 & 0.265 & 49.6 & 550.6 & 9.4 \\
\hline & 2 & 29.26 & 132.6 & 0.0296 & 0.0006 & 3.240 & 99.9 & 0.056 & 132.5 & 1208.0 & 4.9 \\
\hline & 3 & 37.31 & 130.7 & 0.0154 & 0.0002 & 1.040 & 100.0 & 0.029 & 130.6 & 1195.4 & 5.8 \\
\hline & 4 & 100.0 & 131.0 & 0.0378 & 0.0021 & 8.106 & 99.5 & 0.072 & 130.3 & 1193.9 & 4.9 \\
\hline \multirow[t]{4}{*}{5} & 1 & 12.33 & 127.8 & 0.0455 & 0.0093 & 2.002 & 97.9 & 0.087 & 125.0 & 1158.1 & 7.9 \\
\hline & 2 & 28.83 & 133.7 & 0.0068 & 0.0036 & 2.678 & 99.2 & 0.013 & 132.6 & 1209.1 & 12.5 \\
\hline & 3 & 76.79 & 131.9 & 0.0217 & 0.0016 & 7.786 & 99.6 & 0.041 & 131.4 & 1201.0 & 5.4 \\
\hline & 4 & 100.0 & 130.9 & 0.0166 & 0.0016 & 3.768 & 99.6 & 0.032 & 130.4 & 1194.4 & 5.6 \\
\hline
\end{tabular}


Table 2. cont. ${ }^{40} \mathrm{Ar} /{ }^{39} \mathrm{Ar}$ Step-Heating Analytical Results

\begin{tabular}{|c|c|c|c|c|c|c|c|c|c|c|c|}
\hline $\begin{array}{l}\text { Grain } \\
\text { No }\end{array}$ & $\begin{array}{l}\text { Step } \\
\text { No }\end{array}$ & $\begin{array}{l}\text { Cum } \\
\% 0^{39} \mathrm{Ar}\end{array}$ & ${ }^{40} \mathrm{Ar} /{ }^{39} \mathrm{Ar}$ & ${ }^{37} \mathrm{Ar} /{ }^{39} \mathrm{Ar}$ & ${ }^{36} \mathrm{Ar} /{ }^{39} \mathrm{Ar}$ & $\begin{array}{l}\text { Vol. }{ }^{39} \mathrm{Ar} \\
\times 10^{-14} \mathrm{~mol}\end{array}$ & $\begin{array}{c}\text { \%Rad. } \\
{ }^{40} \mathrm{Ar}\end{array}$ & $\mathrm{Ca} / \mathrm{K}$ & ${ }^{40} \mathrm{Ar} * / 39 \mathrm{Ar}$ & $\begin{array}{l}\text { Age } \\
(\mathrm{Ma})\end{array}$ & $\begin{array}{c} \pm 1 \text { s.d. } \\
\text { (Ma) }\end{array}$ \\
\hline \multicolumn{12}{|c|}{ Kuhmo (Lent-W1) } \\
\hline \multicolumn{12}{|c|}{$\mathrm{J}$-value $=0.0071985 \pm 0.0000072$} \\
\hline \multirow[t]{2}{*}{1} & 1 & 35.37 & 135.6 & 0.1530 & 0.0053 & 2.604 & 98.9 & 0.291 & 134.0 & 1218.6 & 3.8 \\
\hline & 2 & 53.14 & 136.5 & 0.0127 & 0.0153 & 3.737 & 96.7 & 0.024 & 132.0 & 1205.0 & 4.3 \\
\hline \multirow[t]{2}{*}{2} & 1 & 67.32 & 131.4 & 0.1437 & 0.0036 & 2.851 & 99.2 & 0.273 & 130.4 & 1194.4 & 4.2 \\
\hline & 2 & 100.0 & 133.3 & 0.0116 & 0.0017 & 4.037 & 99.6 & 0.022 & 132.8 & 1210.2 & 3.6 \\
\hline \multirow[t]{3}{*}{3} & 1 & 16.52 & 129.2 & 0.0368 & 0.0062 & 1.676 & 98.6 & 0.070 & 127.4 & 1174.3 & 2.9 \\
\hline & 2 & 40.34 & 135.5 & 0.0060 & 0.0027 & 2.009 & 99.4 & 0.011 & 134.6 & 1222.5 & 8.6 \\
\hline & 3 & 73.94 & 133.2 & 0.0088 & 0.0024 & 0.941 & 99.5 & 0.017 & 132.4 & 1207.9 & 11.6 \\
\hline \multirow[t]{3}{*}{4} & 1 & 100.0 & 134.4 & 0.0947 & 0.0130 & 1.327 & 97.2 & 0.180 & 130.6 & 1195.6 & 5.9 \\
\hline & 2 & 17.47 & 132.0 & 0.0116 & 0.0013 & 3.757 & 99.7 & 0.022 & 131.6 & 1202.6 & 5.9 \\
\hline & 3 & 48.76 & 132.2 & 0.0365 & 0.0021 & 3.040 & 99.5 & 0.070 & 131.6 & 1202.1 & 3.9 \\
\hline \multirow[t]{2}{*}{5} & 1 & 82.30 & 127.8 & 0.0173 & 0.0030 & 0.358 & 99.3 & 0.033 & 126.9 & 1170.7 & 24.4 \\
\hline & 2 & 100.0 & 131.4 & 0.0022 & 0.0001 & 3.924 & 100.0 & 0.004 & 131.4 & 1201.0 & 7.5 \\
\hline \multirow[t]{3}{*}{6} & 1 & 4.20 & 131.4 & 0.0289 & 0.0037 & 2.205 & 99.2 & 0.055 & 130.3 & 1193.9 & 6.4 \\
\hline & 2 & 29.26 & 131.8 & 0.0031 & 0.0011 & 1.990 & 99.8 & 0.006 & 131.5 & 1201.5 & 5.1 \\
\hline & 3 & 37.31 & 130.3 & 0.0031 & 0.0002 & 2.563 & 100.0 & 0.006 & 130.3 & 1193.5 & 6.1 \\
\hline
\end{tabular}

Kuhmo (DC401 308-328)

$\mathrm{J}$-value $=0.0071985 \pm 0.0000072$

\begin{tabular}{|c|c|c|c|c|c|c|c|c|c|c|c|}
\hline \multirow[t]{3}{*}{1} & 1 & 42.41 & 289.1 & 0.4241 & 0.3219 & 0.869 & 67.1 & 0.806 & 194.0 & 1577.0 & 10.0 \\
\hline & 2 & 67.35 & 244.0 & 0.5428 & 0.0543 & 0.511 & 93.4 & 1.030 & 228.1 & 1752.9 & 20.0 \\
\hline & 3 & 100.00 & 255.6 & 4.8588 & 0.0428 & 0.669 & 95.2 & 9.260 & 244.2 & 1830.1 & 17.2 \\
\hline \multirow[t]{3}{*}{2} & 1 & 43.13 & 348.5 & 2.5045 & 0.4696 & 0.088 & 60.2 & 4.770 & 210.3 & 1662.9 & 67.4 \\
\hline & 2 & 92.92 & 330.0 & 0.6833 & 0.0943 & 0.102 & 91.6 & 1.300 & 302.1 & 2084.2 & 91.5 \\
\hline & 3 & 100.00 & 229.0 & 1.4828 & 0.0592 & 0.015 & 92.4 & 2.820 & 211.9 & 1671.0 & 332.5 \\
\hline \multirow[t]{3}{*}{3} & 1 & 79.99 & 414.6 & 1.6426 & 0.5008 & 0.081 & 64.3 & 3.120 & 267.1 & 1934.9 & 74.3 \\
\hline & 2 & 98.59 & 390.9 & 1.4451 & 0.0822 & 0.019 & 93.8 & 2.750 & 367.2 & 2332.4 & 308.9 \\
\hline & 3 & 100.00 & 486.4 & 3.5962 & 0.6550 & 0.001 & 60.3 & 6.850 & 293.9 & 2050.4 & 2212.7 \\
\hline \multirow[t]{2}{*}{4} & 1 & 69.45 & 315.2 & 1.3051 & 0.2050 & 0.272 & 80.8 & 2.480 & 255.0 & 1880.2 & 39.3 \\
\hline & 2 & 100.00 & 383.2 & 6.9105 & 0.0601 & 0.120 & 95.5 & 13.200 & 367.6 & 2334.1 & 33.5 \\
\hline \multirow[t]{3}{*}{5} & 1 & 70.43 & 280.9 & 0.1149 & 0.1244 & 4.001 & 86.9 & 0.218 & 244.3 & 1830.6 & 5.6 \\
\hline & 2 & 98.31 & 311.1 & 0.0789 & 0.0376 & 1.584 & 96.4 & 0.150 & 300.0 & 2075.5 & 9.1 \\
\hline & 3 & 100.00 & 280.2 & 0.1036 & 0.0414 & 0.096 & 95.6 & 0.197 & 268.1 & 1939.2 & 39.0 \\
\hline \multirow[t]{3}{*}{6} & 1 & 15.52 & 131.2 & 0.1338 & 0.0139 & 1.111 & 96.9 & 0.254 & 127.2 & 1172.6 & 11.0 \\
\hline & 2 & 61.27 & 163.2 & 0.1664 & 0.0009 & 3.275 & 99.8 & 0.316 & 163.0 & 1400.4 & 5.8 \\
\hline & 3 & 100.00 & 160.4 & 0.2372 & 0.0007 & 2.772 & 99.9 & 0.451 & 160.2 & 1383.9 & 7.2 \\
\hline \multirow[t]{3}{*}{7} & 1 & 75.83 & 361.6 & 0.9819 & 0.2697 & 0.248 & 78.0 & 1.870 & 282.2 & 2000.8 & 23.5 \\
\hline & 2 & 98.62 & 424.3 & 0.0934 & 0.0848 & 0.075 & 94.1 & 0.177 & 399.2 & 2443.2 & 57.0 \\
\hline & 3 & 100.00 & 820.1 & 4.2059 & 0.5002 & 0.005 & 82.0 & 8.020 & 674.5 & 3188.6 & 2095.9 \\
\hline \multirow[t]{3}{*}{8} & 1 & 29.94 & 231.0 & 0.0280 & 0.0123 & 2.056 & 98.4 & 0.053 & 227.4 & 1749.3 & 8.8 \\
\hline & 2 & 64.28 & 246.6 & 0.0150 & 0.0035 & 2.358 & 99.6 & 0.028 & 245.5 & 1836.3 & 11.0 \\
\hline & 3 & 100.00 & 243.5 & 0.0197 & 0.0027 & 2.453 & 99.7 & 0.037 & 242.7 & 1823.0 & 8.0 \\
\hline
\end{tabular}

i) Errors are one sigma uncertainties and exclude uncertainties in the J-value.

ii) Data are corrected for mass spectrometer backgrounds, discrimination and radioactive decay.

iii) Interference corrections: $\left({ }^{36} \mathrm{Ar} /{ }^{37} \mathrm{Ar}\right) \mathrm{Ca}=2.83 \mathrm{E}-4 ;\left({ }^{39} \mathrm{Ar} /{ }^{37} \mathrm{Ar}\right) \mathrm{Ca}=6.86 \mathrm{E}-4 ;\left({ }^{40} \mathrm{Ar} /{ }^{39} \mathrm{Ar}\right) \mathrm{K}=1.5 \mathrm{E}-3$

iv) J-value is based on an age of $98.8 \mathrm{Ma}$ for GA-1550 biotite. 
a)

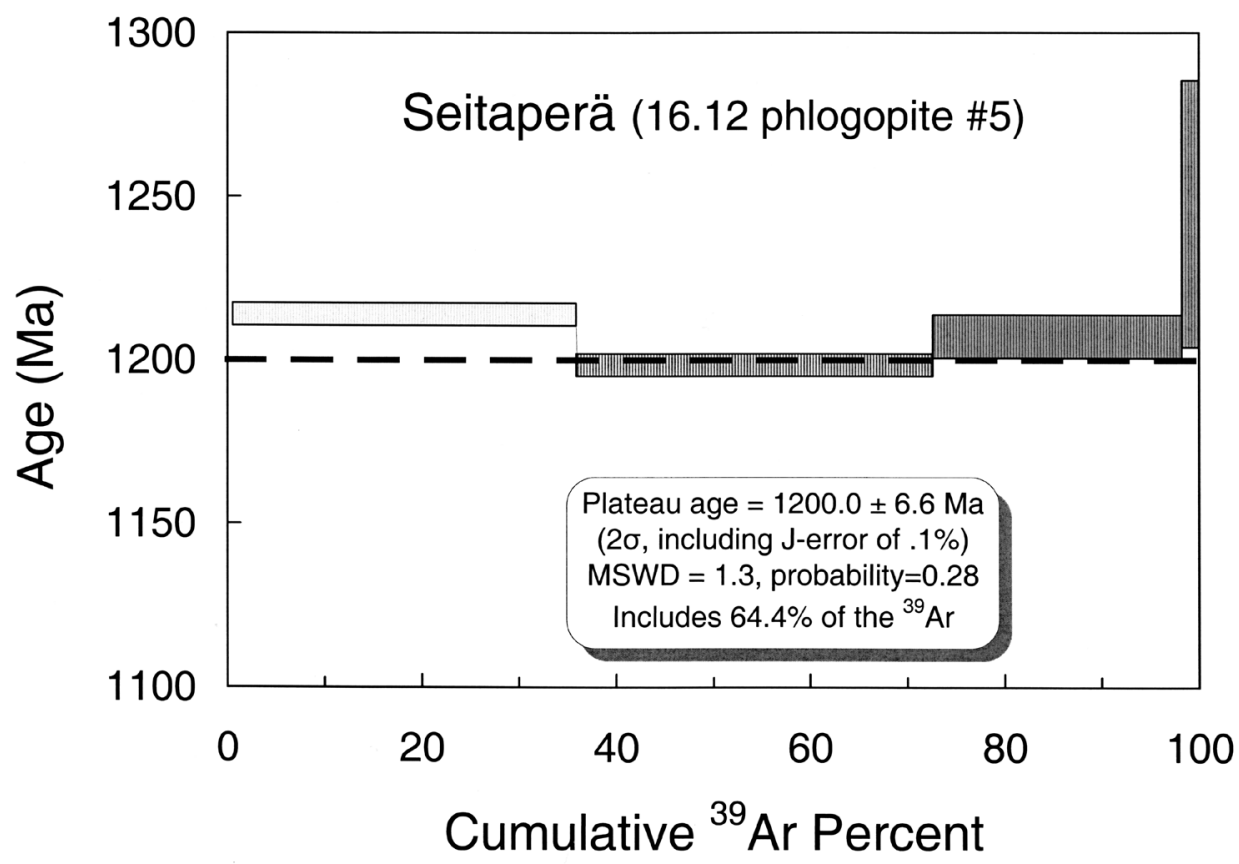

b)

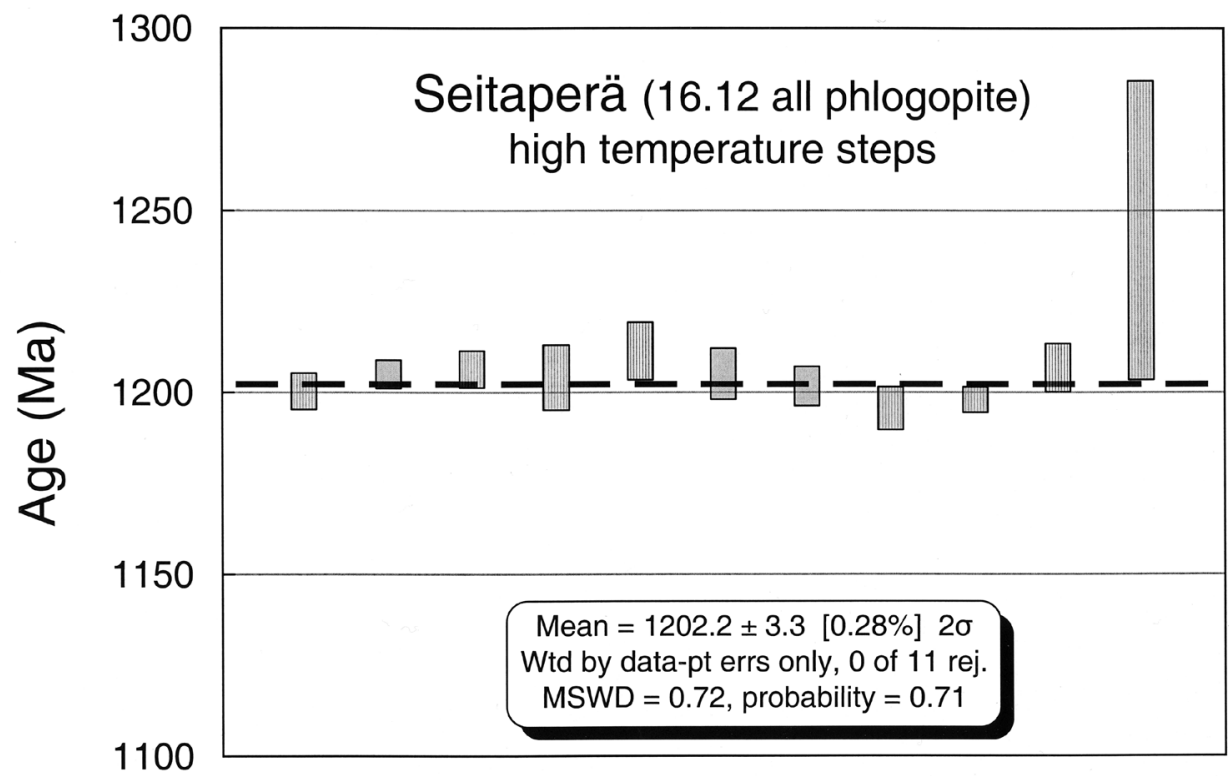

Fig. $3 .{ }^{40} \mathrm{Ar} /{ }^{39} \mathrm{Ar}$ plots for sample 16.12, Seitaperä. a) ${ }^{40} \mathrm{Ar} /{ }^{39} \mathrm{Ar}$ step-heating age spectrum for phlogopite grain \#I from sample 16.12. b) Histogram showing ${ }^{40} \mathrm{Ar} /{ }^{39} \mathrm{Ar}$ ages obtained from the high temperature heating steps for five phlogopite grains, sample 16.12. Error boxes are one-sigma uncertainties. Uncertainties in the weighted mean ages are two-sigma. 
a)

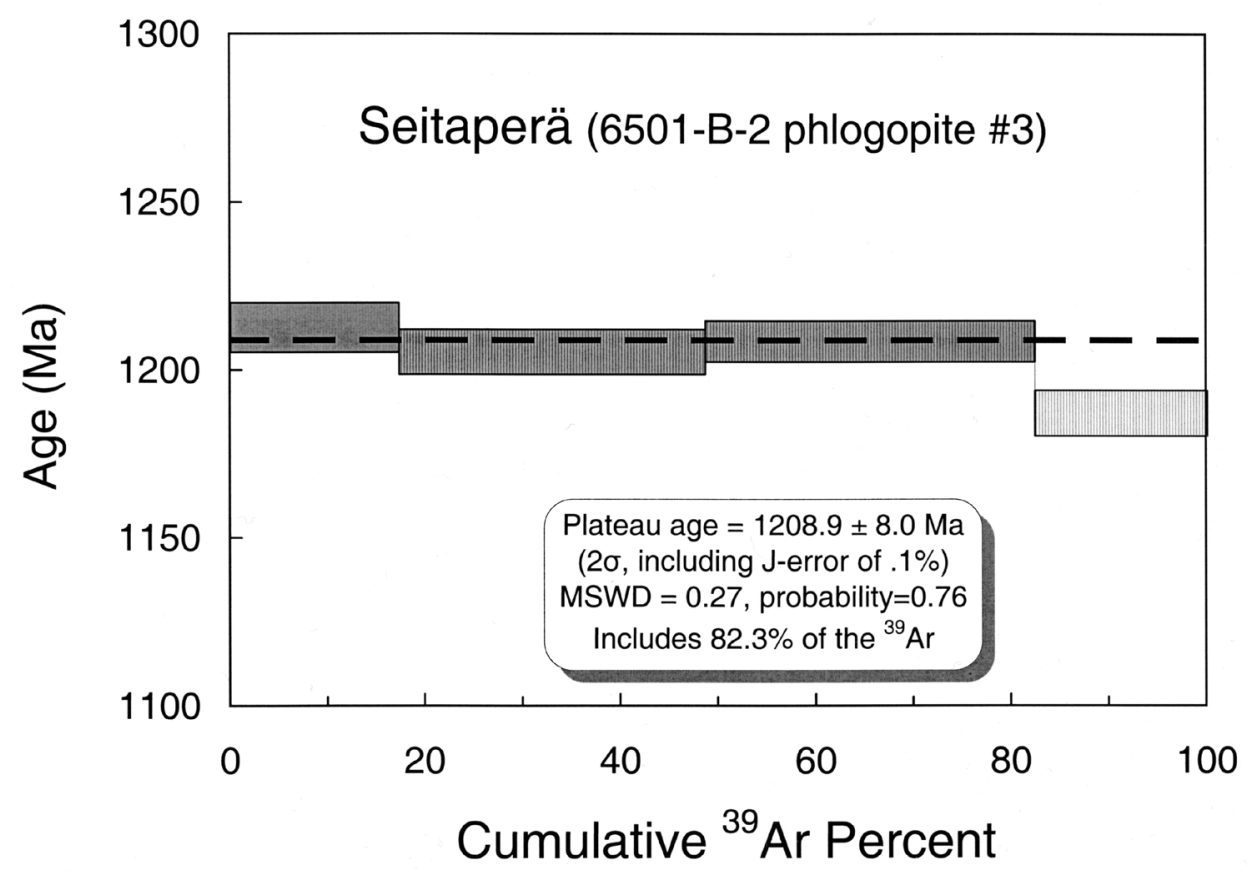

b)

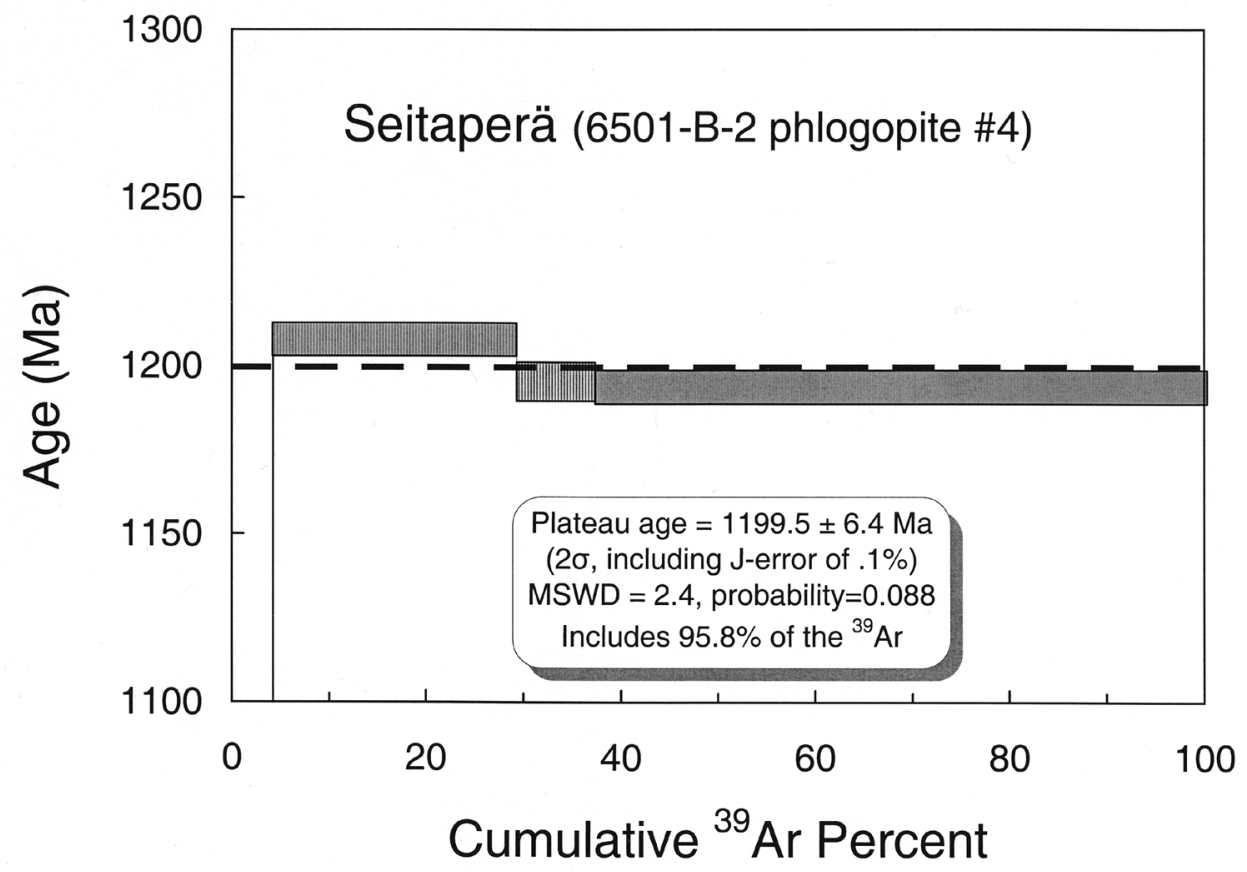

Fig. $4 .{ }^{40} \mathrm{Ar} /{ }^{39} \mathrm{Ar}$ plots for sample $650 \mathrm{I}-\mathrm{B}-2$, Seitaperä. a) ${ }^{40} \mathrm{Ar} /{ }^{39} \mathrm{Ar}$ step-heating age spectrum for phlogopite grain \#3 from sample 650I-B-2. b) ${ }^{40} \mathrm{Ar} /{ }^{39} \mathrm{Ar}$ step-heating age spectrum for phlogopite grain \#4 from sample 650I-B-2. 
c)

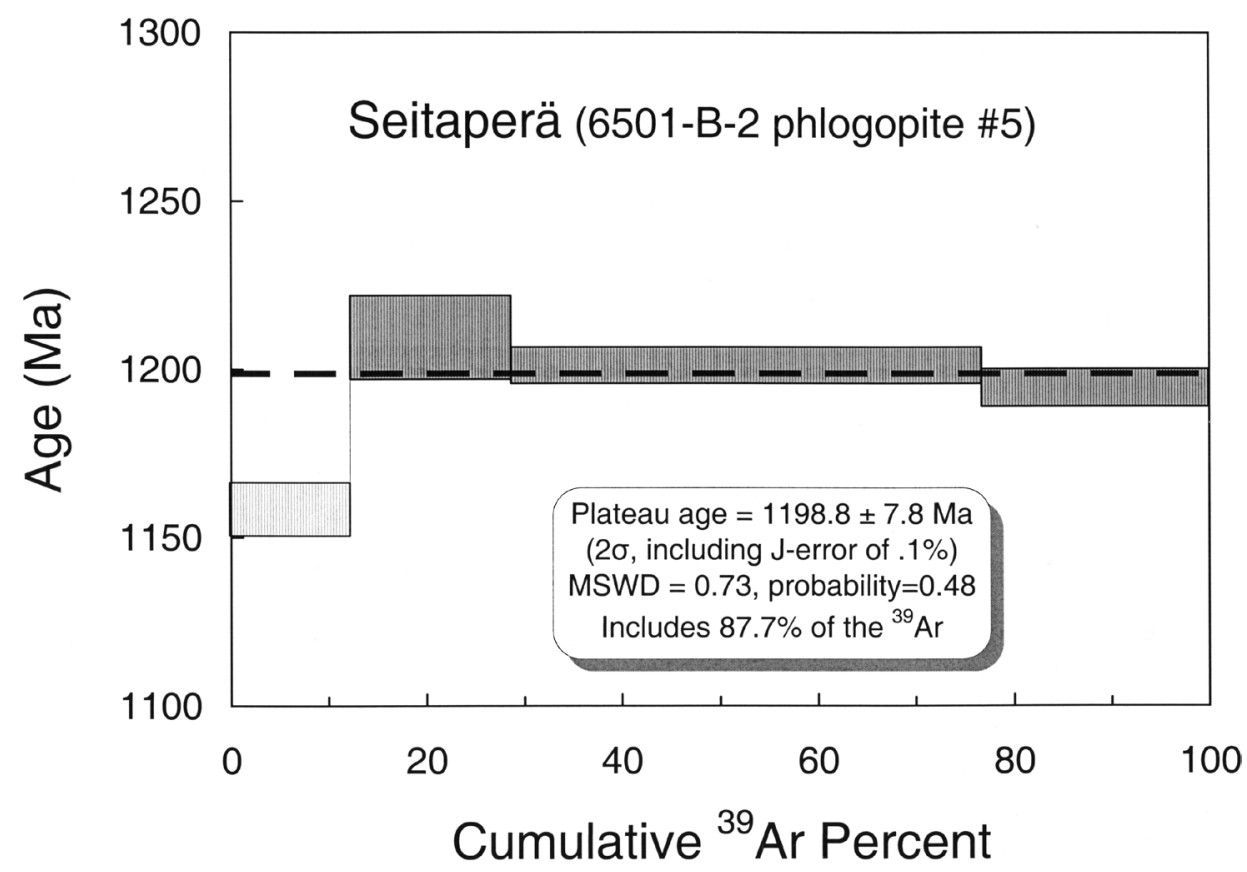

d)

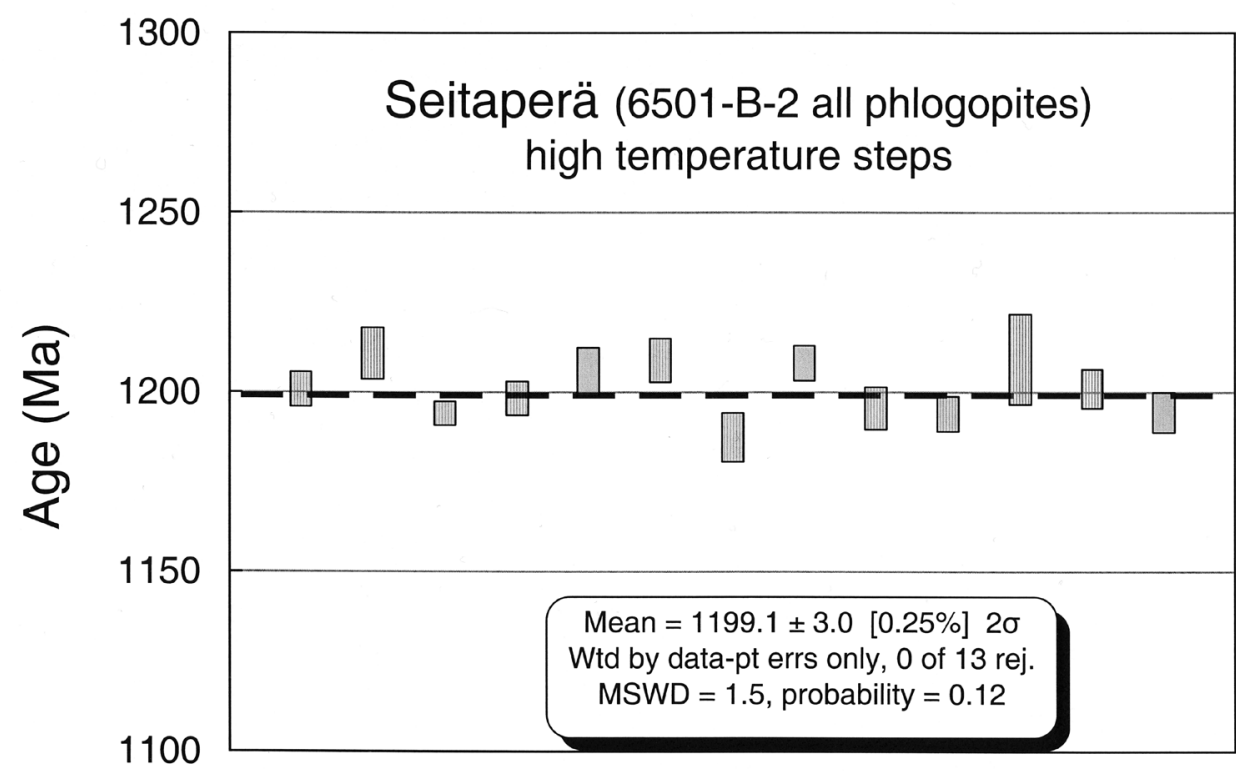

Fig. 4. continued. c) ${ }^{40} \mathrm{Ar} /{ }^{39} \mathrm{Ar}$ step-heating age spectrum for phlogopite grain \#5 from sample $650 \mathrm{I}-\mathrm{B}-2$. d) Histogram showing high temperature ${ }^{40} \mathrm{Ar} /{ }^{39} \mathrm{Ar}$ ages obtained from five phlogopite grains, sample $650 \mathrm{I}-\mathrm{B}-2$. Error boxes are one-sigma uncertainties. Uncertainties in the weighted mean ages are two-sigma errors. 


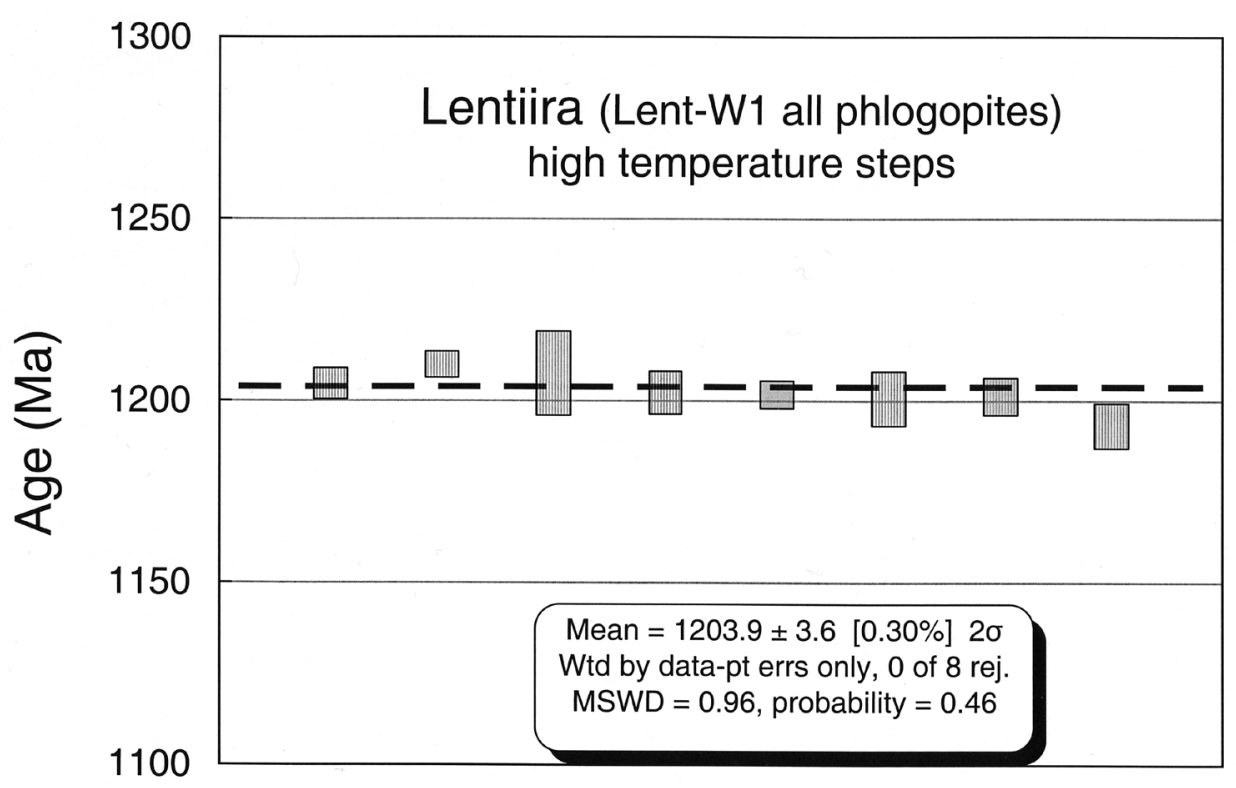

Fig. 5. Histogram showing high temperature ${ }^{40} \mathrm{Ar} /{ }^{39} \mathrm{Ar}$ ages obtained from six phlogopite grains, sample Lent-WI, Lentiira. Error boxes are one-sigma uncertainties. Uncertainties in the weighted mean age are two-sigma errors.

proximation to the time of dike emplacement $(\sim 1.2$ $\mathrm{Ga}$ ), consistent with results from Lent-W1.

\section{Discussion}

As a group the Ar-Ar age data from samples 16.12 $27 \mathrm{~m}, 6501-\mathrm{B}-2$ and Lent-W1 produced ages that are all within error of each other, $1202 \pm 3 \mathrm{Ma}(2 \sigma)$, $1199 \pm 3 \mathrm{Ma}(2 \sigma)$ and $1204 \pm 4 \mathrm{Ma}(2 \sigma)$, respectively. The consistency of the step-heating results suggests that these ages represent precise determinations of the dike emplacement times at these localities. The fact that they overlap in time, combined with their mineralogical and geochemical similarities, suggest that these dikes represent a cogenetic suite of magmas.

The question arises however, are the kimberlitic rocks on the Finnish side of the border time correlative to those in the Kostomuksha area in Russian Karelia? As noted earlier, the Rb-Sr data would suggest not, the Kostomuksha dikes would seem to be roughly $30 \pm 10$ m.y. older than their Finnish counterparts. Yet the data of Wiersburg (2001), although producing rather scattered $\mathrm{K}$-Ar ages, nevertheless center on $1193 \mathrm{Ma}$, and consequently would sug- gest just the opposite, that the two groups are indeed time correlative. The answer to this question can only come from further age determinations on rocks from both sides of the border. Particularly useful would be laser Ar-Ar dating, as was conducted in this study, on a selected suite of fresh samples from the Kostomuksha dikes.

Interestingly, $1200 \mathrm{Ma}$ marks a significant worldwide kimberlite/lamproite intrusive event. The best known include: 1. the major diamond producing Argyle olivine lamproite at $1178 \pm 47 \mathrm{Ma}$ (Pidgeon et al., 1988; Sun et al., 1986), 2. the Krishna lamproites at $1224 \pm 14 \mathrm{Ma}$ (Kumar et al., 2001) thought to be the source rocks of the most famous Indian diamonds, 3. the Premier kimberlite in South Africa at $1198 \pm$ $14 \mathrm{Ma}$ (Phillips et al., 1989) known for producing some of the largest diamonds ever mined. Although not yet proven to contain significant diamonds, the olivine lamproites - Group II kimberlites of LentiiraKuhmo-Kostomuksha may still prove to be economically interesting, as the Archean mantle they have ascended through has all of the hallmarks for good diamond potential (e.g. O'Brien et al., 2003). 


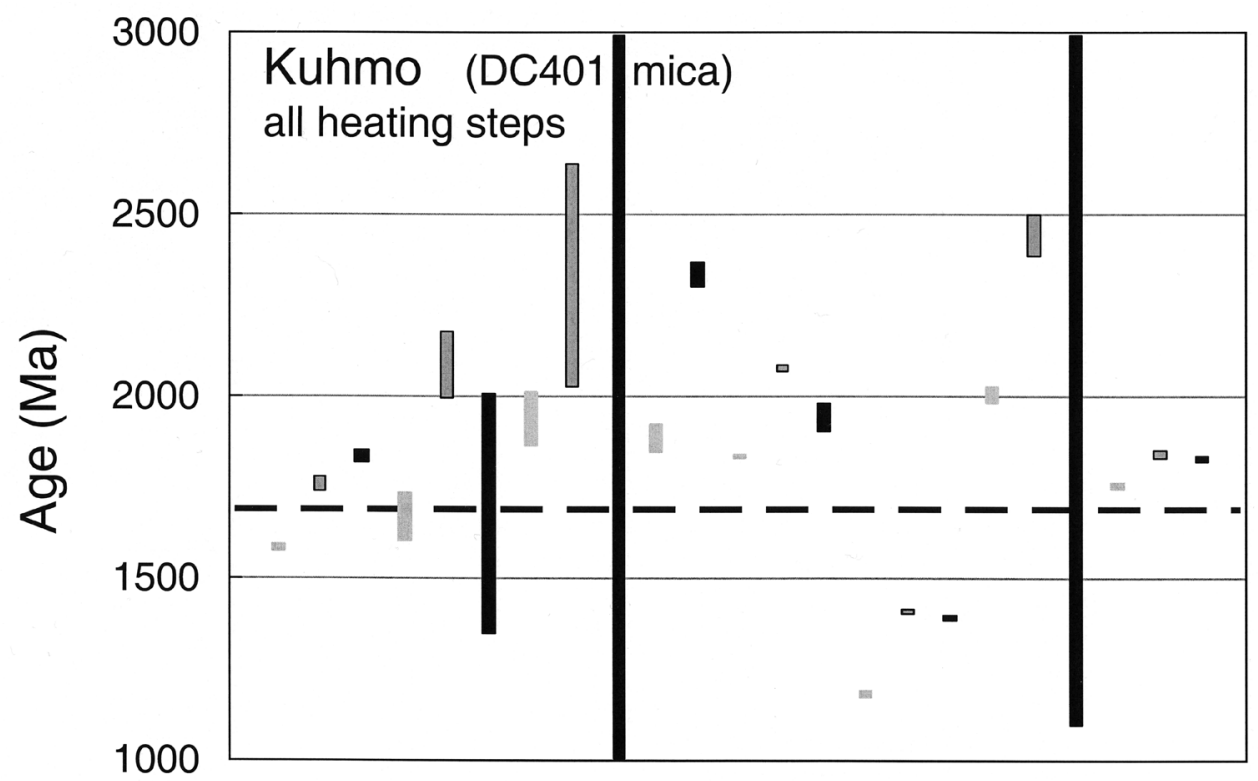

Fig. 6. Histogram showing ${ }^{40} \mathrm{Ar} /{ }^{39} \mathrm{Ar}$ results for eight biotite grains from sample DC40I, Lentiira. Light grey $=$ low temperature step; medium grey $=$ intermediate temperature step; black $=$ high temperature step. Error boxes are one-sigma uncertainties.

\section{Acknowledgements}

The authors would very much like to thank European Diamonds Plc for providing samples of their kimberlite discoveries in West and East Lentiira and deeply appreciate the financial support by De Beers to the Noble Gas Laboratory, the University of Melbourne, which made the $\mathrm{Ar}-\mathrm{Ar}$ analyses reported here possible.

\section{References}

Belyatskii, B.V., Nikitina, L.P., Savva, E.V. \& Levskii, L.K., 1997. Isotopic signatures of lamproite dikes on the Eastern Baltic shield. Geochemistry International 35, 575579.

Kumar, A., Gopalan, K., Rao, K.R.P. \& Nayak, S.S., 2001. $\mathrm{Rb}-\mathrm{Sr}$ age of kimberlites and lamproites from Eastern Dharwar craton, South India. Journal of the Geological Society of India 58, 135-141.
Lehtonen, M. 2005. Kimberlites in Finland: information about the mantle of the Karelian Craton and implications for diamond exploration. Geological Survey of Finland, Espoo, Academic Dissertation 31, 82 p.

Ludwig, K.R., 2000. User's Manual for Isoplot/Ex v.2.2 a Geochronological Toolkit for Microsoft Excel.

Mahotkin, I.L., 1998. Petrology of Group 2 Kimberlite-Olivine lamproite (K2L) series from the Kostomuksha area, Karelia, N.W. Russia. In: Gurney, J.J. et al. (eds.) $7^{\text {th }}$ International Kimberlite Conference, Extended Abstracts Volume, Cape Town, South Africa, pp. 529-531.

McDougall, I. \& Harrison, T.M., 1999. Geochronology and thermochronology by the ${ }^{40} \mathrm{Ar} /{ }^{39} \mathrm{Ar}$ method. Second Edition. Oxford University Press, New York, 288 p.

Nikitina, L.P., Levsky, L.K., Lohkov, K.I., Belyatsky, B.V., Zhuravlev, V.A., Lepekhina, E.N. \& Antontov, A.V., 1999. Proterozoic alkaline-ultramafic magmatism in the eastern part of the Baltic Shield. Petrology 7, 246-266.

O'Brien, H.E. \& Tyni, M., 1999. Mineralogy and geochemistry of kimberlites and related rocks from Finland. In: 
Gurney, J.J. et al. (eds.) Proceedings of the $7^{\text {th }}$ International Kimberlite Conference, Cape Town, South Africa, pp. 625-636.

O'Brien, H.E., Lehtonen, M., Spencer, R. \& Birnie, A., 2003. Lithospheric mantle in Eastern Finland: A 250 $\mathrm{km} 3 \mathrm{D}$ transcect. In: Extended Abstracts of the $8^{\text {th }}$ International Kimberlite Conference, Victoria, Canada, FLA_0261, 5 p.

O'Brien, H.E., Peltonen, P. \& Vartianen, H., 2005. Kimberlites, carbonatites, and alkaline rocks. In: Lehtinen, M. et al. (eds.) Precambrian geology of Finland - Key to the evolution of the Fennoscandian Shield. Developments in Precambrian geology 14. Elsevier B.V., Amsterdam, pp. 605-644.

Phillips, D., Onstott, T.C. \& Harris, J.W., 1989. ${ }^{40} \mathrm{Ar} /{ }^{39} \mathrm{Ar}$ laser-probe dating of diamond inclusions from the Premier kimberlite. Nature 340, 460-462.

Pidgeon R.T., Smith, C.B. \& Fanning, C.M., 1988. Kimberlite and lamproite emplacement ages in Western Australia. Proceedings of the $4^{\text {th }}$ International Kimberlite Conference, Geological Society of Australia, Special Publication 14, 369-381.

Renne P., Swisher C.C., Deino A.L., Karner D.BV., Owens T.L. \& DePaolo D.J., 1998. Intercalibration of standards, absolute ages and uncertainties in ${ }^{40} \mathrm{Ar} /{ }^{39} \mathrm{Ar}$ dating. Chemical Geology 145, 117-152.

Steiger, R.H. \& Jager, E., 1977. Subcommission on geochronology: Convention on the use of decay constants in geo- and cosmochronology. Earth and Planetary Science Letters 36, 359-362.

Sun, S.S., Jaques, A.L. \& McCulloch, M.T., 1986, Isotopic evolution of the Kimberley Block, Western Australia. In: Extended Abstracts of the $4^{\text {th }}$ International Kimberlite Conference, Perth, Australia, Geological Society of Australia Abstracts 16, 346-348.

Wiersberg, T., 2001. Edelgase als Tracer für Wechselwirkungen von Krusten- und Mantelfluiden mit diamantführenden Gesteinen des östlichen Baltischen Schildes. Ph.D. Dissertation, University of Postdam, 113 p.

Wiersberg, T., Niedermann, S., Erzinger, J., Levsky, Lev K, $\&$ Lokhov, K. I., 1999. Noble gas investigations of ancient subcontinental mantle samples (kimberlites and lamproites) from the eastern Baltic shield. EUG 10, Journal of Conference Abstracts 4, p. 372.

Wiersberg, T., Niedermann, S., Erzinger, J., Levsky, L. K. \& Lohkov, K.I., 2000. Noble gas investigations of Early Proterozoic lamproites from the Eastern Baltic Shield. Goldschmidt 2000, Journal of Conference Abstracts 5, p. 1089. 